TRANSACTIONS OF THE

AMERICAN MATHEMATICAL SOCIETY

Volume 364, Number 1, January 2012, Pages 175-194

S 0002-9947(2011)05349-3

Article electronically published on August 31, 2011

\title{
THE CLASSIFICATION OF TORSION-FREE ABELIAN GROUPS OF FINITE RANK UP TO ISOMORPHISM AND UP TO QUASI-ISOMORPHISM
}

\author{
SAMUEL COSKEY
}

\begin{abstract}
The isomorphism and quasi-isomorphism relations on the $p$-local torsion-free abelian groups of rank $n \geq 3$ are incomparable with respect to Borel reducibility.
\end{abstract}

\section{INTRODUCTION}

This paper extends some recent work (by Hjorth, Kechris, Adams and Thomas) on the complexity of the classification problem for torsion-free abelian groups of finite rank. In 1937, Baer solved the classification problem for torsion-free abelian groups of rank 1. The rank 2 groups resisted satisfactory classification for sixty years, after which Hjorth Hjo99 used the theory of countable Borel equivalence relations to prove that the classification problem for rank 2 torsion-free abelian groups is genuinely more complex than that for rank 1 torsion-free abelian groups. Building upon the work of Adams-Kechris AK00, Thomas Tho03a later proved that the complexity of the classification problem for torsion-free abelian groups of rank $n$ strictly increases with $n$.

As a stepping stone to this result, Thomas Tho03a proved that the classification problem for torsion-free abelian groups of rank $n$ up to quasi-isomorphism strictly increases in complexity with $n$. Here, $A$ and $B$ are said to be quasi-isomorphic iff $A$ is commensurable with an isomorphic copy of $B$. This left open the question of which of the two classification problems for torsion-free abelian groups of fixed rank $n$ is more complex: that up to isomorphism or that up to quasi-isomorphism. In this paper, we prove that if $n \geq 3$, then the two problems have incomparable complexities.

In order to state these results more formally, we must use the terminology of Borel equivalence relations. The idea, due to Friedman-Stanley FS89] and HjorthKechris [HK96], is that often a "classification problem" may be regarded as an equivalence relation on a standard Borel space. (A standard Borel space is a separable, completely metrizable space equipped only with its $\sigma$-algebra of Borel sets.) For instance, any torsion-free abelian group of rank $n$ is isomorphic to a subgroup of $\mathbb{Q}^{n}$. Hence, any torsion-free abelian group of rank $n$ can be presented as an element

Received by the editors February 6, 2009 and, in revised form, March 16, 2010.

2010 Mathematics Subject Classification. Primary 03E15; Secondary 20K15.

Key words and phrases. Countable Borel equivalence relations, torsion-free abelian groups, superrigidity.

This is a part of the author's doctoral thesis, which was written under the supervision of Simon Thomas. This work was partially supported by NSF grant DMS 0600940.

(C)2011 American Mathematical Society 
of the standard Borel space $R(n)$ of all subgroups of $\mathbb{Q}^{n}$ that contain a basis of $\mathbb{Q}^{n}$. Studying the complexity of the classification problem for torsion-free abelian groups of rank $n$ thus amounts to studying the complexity of the isomorphism relation $\cong_{n}$ on $R(n)$.

The equivalence relations $\cong_{n}$ lie in the class of countable Borel equivalence relations, which we now describe. An equivalence relation $E$ on a standard Borel space $X$ is called Borel iff it is a Borel subset of the product space $X \times X$, and it is called countable iff all of its equivalence classes are countable. If $\Gamma$ is a countable group of Borel bijections of $X$, then the corresponding orbit equivalence relation defined by

$$
x E_{\Gamma} y \Longleftrightarrow x, y \text { lie in the same } \Gamma \text {-orbit }
$$

is easily seen to be a countable Borel equivalence relation. For instance, it is easy to verify that subgroups $A, B \leq \mathbb{Q}^{n}$ are isomorphic iff there exists $g \in \mathrm{GL}_{n}(\mathbb{Q})$ such that $B=g(A)$. Hence, $\cong_{n}$ is the orbit equivalence relation induced by the action of $\mathrm{GL}_{n}(\mathbb{Q})$ on $R(n)$, and thus it is a countable Borel equivalence relation.

In fact, by Feldman and Moore [FM77, any countable Borel equivalence relation on a standard Borel space $X$ arises as the orbit equivalence relation induced by a Borel action of a countable group $\Gamma$ on $X$. We remark that neither the group $\Gamma$ nor its action is canonically determined by $E_{\Gamma}$; the case of $\cong_{n}$ is special in the sense that there is a natural group action which induces it.

We now discuss how to compare the complexity of two equivalence relations. If $E, F$ are equivalence relations on the standard Borel spaces $X, Y$, respectively, then we write $E \leq_{B} F$ and say that $E$ is Borel reducible to $F$ iff there exists a Borel function $f: X \rightarrow Y$ such that for all $x, x^{\prime} \in X$,

$$
x E x^{\prime} \Longleftrightarrow f(x) F f\left(x^{\prime}\right) .
$$

The relationship $E \leq_{B} F$ means that elements of $X$ can be explicitly classified up to $E$ using invariants from the quotient space $Y / F$, considered with its quotient Borel structure. Additionally, $E \leq_{B} F$ implies that, structurally, $X / E$ is a simpler space of invariants than $Y / F$.

Extending this notation in the obvious fashion, we write $E \sim_{B} F$ when both $E \leq_{B} F$ and $F \leq_{B} E$, we write $E<_{B} F$ when both $E \leq_{B} F$ and $E \chi_{B} F$, and we write $E \perp_{B} F$ when both $E \mathbb{Z}_{B} F$ and $F \mathbb{Z}_{B} E$. Returning to torsion-free abelian groups, we can now state Hjorth's and Thomas's aforementioned theorems together as:

$$
\cong_{1}<_{B} \cong_{2}<_{B} \cong_{3}<_{B} \cdots<_{B} \cong_{n}<_{B} \cdots
$$

This was the first naturally occurring example of an infinite $\leq_{B}$-chain. Shortly after this was found, Thomas found an infinite $\leq_{B}$-antichain, again consisting of the isomorphism relations on various spaces of torsion-free abelian groups of finite rank. If $p$ is prime, the abelian group $A$ is said to be $p$-local iff it is infinitely $q$-divisible for every prime $q \neq p$. Let $R(n, p)$ denote the subspace of $R(n)$ consisting of just the $p$-local torsion-free abelian groups of rank $n$, and let $\cong_{n, p}$ denote the restriction of $\cong_{n}$ to $R(n, p)$. Thomas's theorem says that if $n \geq 3$ and $p, q$ are distinct primes, then $\cong_{n, p}$ is Borel incomparable with $\cong_{n, q}$. (This was later extended to include the case $n=2$ by Hjorth-Thomas [HT06.)

In our comparison of isomorphism and quasi-isomorphism, we shall consider only the $p$-local groups. It will actually be necessary to restrict our attention to a slightly smaller space, that of $p$-local torsion-free abelian groups of fixed divisible 
rank. Here, the divisible rank of a (finite rank) torsion-free abelian group $A$ is defined as the maximum $k$ of the ranks of the divisible quotients of $A$. We let $R(n, p, k)$ denote the subspace of $R(n, p)$ consisting of just the $p$-local torsion-free abelian groups of rank $n$ and of divisible rank $k$. Let $\cong_{n, p}^{k}$ denote the restriction of $\cong_{n}$ to $R(n, p, k)$.

Theorem A. Let $n \geq 3$ and $p$ be a prime, and suppose that $k, l<n$ and $k \neq l$. Then $\cong_{n, p}^{k}$ is Borel incomparable with $\cong_{n, p}^{l}$.

We now turn to a comparison of the isomorphism and quasi-isomorphism relations on $R(n, p, k)$. Recall that torsion-free abelian groups $A, B \leq \mathbb{Q}^{n}$ are said to be quasi-isomorphic iff $A$ is commensurable with an isomorphic copy of $B$ (i.e., there exists $B^{\prime} \cong B$ such that $A \cap B^{\prime}$ has finite index in $A$ and in $\left.B^{\prime}\right)$. Let $\sim_{n, p}^{k}$ denote the quasi-isomorphism equivalence relation on $R(n, p, k)$. Thomas found the quasi-isomorphism relation easier to work with in Tho03a, for reasons which will become clear later on in this paper. However, the next theorem shows that the classification of ( $p$-local) torsion-free abelian groups up to quasi-isomorphism is not simpler than that up to isomorphism.

Theorem B. If $1 \leq k \leq n-2$, then $\cong_{n, p}^{k}$ is Borel incomparable with $\sim_{n, p}^{k}$.

It should be noted that by [Tho02, Theorem 4.4], when $k=n-1$, the notion of quasi-isomorphism coincides with that of isomorphism.

It follows easily from Theorems A and B, together with [Tho02, Theorem 4.7], that for $n \geq 3$, the isomorphism and quasi-isomorphism relations on the space of all local (that is, $p$-local for some $p$ ) torsion-free abelian groups of rank $n$ are also incomparable.

Conjecture. For $n \geq 3$, the isomorphism and quasi-isomorphism relations on the space $R(n)$ of all torsion-free abelian groups of rank $n$ are Borel incomparable.

This paper is organized as follows. In the second section, we introduce ergodic theory and homogeneous spaces. Our main example is the $k$-Grassmann space $\operatorname{Gr}_{k}\left(\mathbb{Q}_{p}^{n}\right)$ of $k$-dimensional subspaces of the $n$-dimensional vector space $\mathbb{Q}_{p}^{n}$ over the $p$-adics. We then prove Theorem 2.4, which gives a characterization of certain action-preserving maps between the $k$-Grassmann spaces. In the third section, we state a cocycle superrigidity theorem of Adrian Ioana, and derive Corollary 3.3. which roughly states that a homomorphism of $\mathrm{SL}_{n}(\mathbb{Z})$-orbits between Grassmann spaces is a slight perturbation of an action-preserving map. We then combine Theorem 2.4 and Corollary 3.3 to prove the auxiliary result that for $l \neq n-k$, the orbit equivalence relations induced by the action of $\mathrm{GL}_{n}(\mathbb{Q})$ on $\mathrm{Gr}_{k}\left(\mathbb{Q}_{p}^{n}\right)$ and on $\operatorname{Gr}_{l}\left(\mathbb{Q}_{p}^{n}\right)$ are Borel incomparable. In the fourth section, we use the Kurosh-Malcev completion of torsion-free abelian groups $A \leq \mathbb{Q}^{n}$ to establish a connection (Lemma 4.1) between the space of $p$-local torsion-free abelian groups and the Grassmann spaces. In the last section, we put Theorem 2.4. Corollary 3.3, and Lemma 4.1 together to prove Theorems A and B.

\section{ERgodic theory of homogeneous spaces}

In this section, we define the notion of ergodicity of a measure-preserving action, which plays an essential role in the theory of countable Borel equivalence relations. We then consider the case of countable groups $\Gamma$ acting on homogeneous spaces for 
compact $K$ such that $\Gamma \leq K$ (by "homogeneous", we simply mean that $K$ acts transitively). As an example, we introduce the Grassmann space of $k$-dimensional subspaces of $\mathbb{Q}_{p}^{n}$. The material of this section is self-contained, but we shall see later that there is a close relationship between Grassmann spaces and spaces of $p$-local torsion-free abelian groups.

Ergodicity and Borel reductions. Let $\Gamma$ be a countable group acting in a Borel fashion on the standard Borel space $X$. If $X$ carries a Borel probability measure $\mu$, then we write $\Gamma \curvearrowright(X, \mu)$ to indicate that $\Gamma$ acts on $X$ in a $\mu$-preserving fashion. (When $\mu$ is clear from the context, we often write $\Gamma \curvearrowright X$.) As before, we let $E_{\Gamma}$ denote the orbit equivalence relation on $X$ induced by the action of $\Gamma$. We say that the action $\Gamma \curvearrowright(X, \mu)$ is ergodic iff every $\Gamma$-invariant subset of $X$ is null or conull for $\mu$. We shall use the characterization that $\Gamma \curvearrowright(X, \mu)$ is ergodic iff for every $\Gamma$-invariant function $f: X \rightarrow Y$ into a standard Borel space $Y$, there exists a conull $A \subset X$ such that $\left.f\right|_{A}$ is a constant function.

This characterization leads to an important generalization of ergodicity which will arise in our arguments. First, if $E, F$ be equivalence relations on standard Borel spaces $X, Y$, we define that a function $f: X \rightarrow Y$ is a Borel homomorphism $f$ from $E$ to $F$ iff

$$
x E y \Longrightarrow f(x) F f(y) .
$$

(This corresponds to using $Y / F$ as a space of incomplete invariants for the $E$ classification problem on $X$.) By the last paragraph, $\Gamma \curvearrowright(X, \mu)$ is ergodic iff every Borel homomorphism from $E_{\Gamma}$ to $I d_{Y}$ is constant on a conull set, where $I d_{Y}$ represents the equality relation on the standard Borel space $Y$. More generally, if $F$ is a Borel equivalence relation on $Y$, then we say $\Gamma \curvearrowright(X, \mu)$ is $F$-ergodic iff for every Borel homomorphism $f$ from $E_{\Gamma}$ to $F$, there exists a $\mu$-conull subset $A \subset X$ such that $f(A)$ is contained in a single $F$-class.

A countable-to-one Borel homomorphism from $E$ to $F$ is called a weak Borel reduction from $E$ to $F$. We write $E \leq_{B}^{w} F$ if there exists a weak Borel reduction from $E$ to $F$. We shall use the fact that if $E, F$ are countable Borel equivalence relations and $\mu$ is nonatomic, then

$$
E \text { is } F \text {-ergodic } \Longrightarrow E \not_{B}^{w} F \Longrightarrow E \mathbb{Z}_{B} F \text {. }
$$

For the first implication, suppose that $E$ is $F$-ergodic and $f$ is a weak Borel reduction from $E$ to $F$. Then there exists a conull subset $M \subset \operatorname{dom} E$ such that $f(M)$ is contained in a single $F$-class. Since $E$ and $F$ are countable and $f$ is countableto-one, it follows that $M$ is a countable conull set, contradicting the fact that $\mu$ is nonatomic. The second implication of (2.1) is clear from the definitions.

Ergodic components. If $\Gamma \curvearrowright(X, \mu)$ is ergodic and $\Lambda \leq \Gamma$ is a subgroup of finite index, then there exists a partition $X=Z_{1} \sqcup \cdots \sqcup Z_{N}$ of $X$ into $\Lambda$-invariant subsets such that for each $i$ :

○ $\mu\left(Z_{i}\right)>0$, and

- $\Lambda \curvearrowright\left(Z_{i}, \mu_{i}\right)$ is ergodic, where $\mu_{i}$ denotes the (normalized) probability measure induced on $Z_{i}$ by $\mu$.

The $\Lambda$-spaces $Z_{i}$ are called the ergodic components for the action $\Gamma \curvearrowright(X, \mu)$. The set of ergodic components is determined uniquely up to null sets by the inclusion of $\Lambda$ into $\Gamma$ and the action of $\Gamma$ on $(X, \mu)$. 
Homogeneous spaces. Let $K$ be a compact, second countable group. If $K$ acts continuously and transitively on the standard Borel space $X$, then $X$ is said to be a homogeneous space for $K$. Every homogeneous space for $K$ is thus in $K$-preserving bijection with $K / L$ for some closed subgroup $L \leq K$. Since $K / L$ carries a unique $K$-invariant probability measure (the projection of the Haar probability measure on $K$ ), it follows that $X$ does as well. Now if $\Gamma \leq K$ is a countable dense subgroup, then the action of $\Gamma$ on $K / L$ clearly preserves the Haar measure, and moreover it is uniquely ergodic with respect to the Haar measure. (Here, the action $\Gamma \curvearrowright Y$ is said to be uniquely ergodic iff there is a unique $\Gamma$-invariant probability measure on $Y$.) It is easy to see that unique ergodicity implies ergodicity, and so $\Gamma \curvearrowright K / L$ is ergodic.

Next, suppose that $\Lambda \leq \Gamma$ is a subgroup of finite index. Then the ergodic components for the action of $\Lambda$ are precisely the orbits of $\bar{\Lambda}$ (the closure in $K$ ) on $K / L$ and each ergodic component is again a homogeneous space for the compact group $K_{0}=\bar{\Lambda}$. If $\Lambda \unlhd \Gamma$ is a normal subgroup of finite index, then $\Gamma$ acts as a transitive permutation group on the $\bar{\Lambda}$-orbits, i.e., on the ergodic components for $\Lambda$. (For proofs of the last few claims, see [Tho03b, Lemma 2.2].)

Example: Grassmann spaces. Let $n$ be a natural number and $p$ a prime. Denote by $\mathbb{Q}_{p}^{n}$ the canonical $n$-dimensional vector space over the field of $p$-adic numbers. Then the $k$-Grassmann space of $\mathbb{Q}_{p}^{n}$, denoted $\operatorname{Gr}_{k}\left(\mathbb{Q}_{p}^{n}\right)$, is the set of $k$-dimensional subspaces of $\mathbb{Q}_{p}^{n}$. Since the compact group $\mathrm{SL}_{n}\left(\mathbb{Z}_{p}\right)$ acts transitively on $\operatorname{Gr}_{k}\left(\mathbb{Q}_{p}^{n}\right)$ Tho03b, Proposition 6.1], we can view $\operatorname{Gr}_{k}\left(\mathbb{Q}_{p}^{n}\right)$ as a homogeneous $\mathrm{SL}_{n}\left(\mathbb{Z}_{p}\right)$-space. Accordingly, it carries a corresponding Haar probability measure and the dense subgroup $\mathrm{SL}_{n}(\mathbb{Z}) \leq \mathrm{SL}_{n}\left(\mathbb{Z}_{p}\right)$ acts (uniquely) ergodically on $\mathrm{Gr}_{k}\left(\mathbb{Q}_{p}^{n}\right)$.

We will be especially interested in the ergodic components of Grassmann space corresponding to the action of the principle congruence subgroups of $\mathrm{SL}_{n}(\mathbb{Z})$. Recall that for any natural number $m$, the principal congruence subgroup $\Gamma_{m} \unlhd \mathrm{SL}_{n}(\mathbb{Z})$ is defined by

$$
\Gamma_{m}=\operatorname{ker}\left[\mathrm{SL}_{n}(\mathbb{Z}) \rightarrow \mathrm{SL}_{n}(\mathbb{Z} / m \mathbb{Z})\right]
$$

where the map on the right-hand side is the canonical surjection. It is easily seen that the closure in $\mathrm{SL}_{n}\left(\mathbb{Z}_{p}\right)$ of $\Gamma_{m}$ is exactly $K_{m}$, where

$$
K_{m}=\operatorname{ker}\left[\mathrm{SL}_{n}\left(\mathbb{Z}_{p}\right) \rightarrow \mathrm{SL}_{n}\left(\mathbb{Z}_{p} / m \mathbb{Z}_{p}\right)\right] .
$$

Hence, the ergodic components of $\mathrm{Gr}_{k}\left(\mathbb{Q}_{p}^{n}\right)$ corresponding to the action of $\Gamma_{m}$ are precisely the $K_{m}$-orbits (for example, see [Tho03b, Lemma 2.2]). As an abbreviation, we shall call these orbits the $\left(m^{\text {th }}\right)$ principle congruence components of the $k$-Grassmann space.

For example, any $V$ in the $K_{p^{t}}$ orbit of $V_{0}:=\mathbb{Q}_{p} e_{1} \oplus \cdots \oplus \mathbb{Q}_{p} e_{k}$ can be written as the column space of a matrix $\left[\begin{array}{l}a \\ b\end{array}\right]$, where $a$ is congruent to the $k \times k$ identity matrix $I_{k}$ modulo $p^{t}$, and $v$ is congruent to 0 modulo $p^{t}$. Since $a$ is clearly invertible, one can use column operations to suppose that $a=I_{k}$. So we have

$$
\left(K_{p^{t}}\right) V_{0}=\left\{\operatorname{col}\left[\begin{array}{c}
I_{k} \\
v
\end{array}\right]: p^{t} \mid v\right\}
$$

where $p^{t} \mid v$ means that for each entry $x$ of $v$, we have that $x / p^{t}$ lies in $\mathbb{Z}_{p}$.

Recall that for $n \geq 3, \mathrm{SL}_{n}(\mathbb{Z})$ has the congruence subgroup property, meaning that every subgroup of finite index contains a principle congruence subgroup. With this, it is easy to derive the following. 
2.3. Proposition. If $n \geq 3$, any ergodic component for the action of a subgroup $\Gamma \leq \mathrm{SL}_{n}\left(\mathbb{Z}_{p}\right)$ of finite index on $\mathrm{Gr}_{k}\left(\mathbb{Q}_{p}^{n}\right)$ contains a principle congruence component.

We close this section with a characterization of the action-preserving maps between ergodic components of Grassmann spaces. In what follows, when $\Gamma \curvearrowright X$ and $\Lambda \curvearrowright Y$, we shall use the term permutation group homomorphism for a pair $(\phi, f)$ where $\phi: \Gamma \rightarrow \Lambda$ is a group homomorphism and $f: X \rightarrow Y$ is a Borel map satisfying $f(\gamma x)=\phi(\gamma) f(x)$ for all $\gamma \in \Gamma$ and $x \in X$.

2.4. Theorem. Let $n \geq 3$ and suppose that $k, l \leq n$. Let $\Gamma_{0}, \Gamma_{1}$ be subgroups of $\mathrm{SL}_{n}(\mathbb{Z})$ of finite index, $X_{0}$ an ergodic component for the action of $\Gamma_{0}$ on $\operatorname{Gr}_{k}\left(\mathbb{Q}_{p}^{n}\right)$, and $X_{1}$ an ergodic component for the action of $\Gamma_{1}$ on $\operatorname{Gr}_{l}\left(\mathbb{Q}_{p}^{n}\right)$. Suppose that:

- $\phi: \Gamma_{0} \rightarrow \Gamma_{1}$ is an isomorphism,

○ $f: X_{0} \rightarrow X_{1}$ is a Borel function, and

○ $(\phi, f): \Gamma_{0} \curvearrowright X_{0} \longrightarrow \Gamma_{1} \curvearrowright X_{1}$ is a permutation group homomorphism.

Then $l=k$ or $l=n-k$, and:

(a) If $l=k \neq n-k$, then there exists $h \in \mathrm{GL}_{n}(\mathbb{Q})$ such that $f$ satisfies $f(x)=h x$ for almost every $x \in X_{0}$.

(b) If $l=n-k \neq k$, then there exists $h \in \mathrm{GL}_{n}(\mathbb{Q})$ such that $f$ satisfies $f(x)=h x^{\perp}$ for almost every $x \in X_{0}$, where $x^{\perp}$ denotes the orthogonal complement of $x$ with respect to the usual dot product.

(c) If $l=k=n / 2$, then either the conclusion of (a) holds or the conclusion of (b) holds.

In the proof, we shall make use of the following well-known result.

2.5. Lemma. Let $n \geq 3$ and $\Gamma_{0} \leq \mathrm{SL}_{n}(\mathbb{Z})$ be a subgroup of finite index. Let $\phi: \Gamma_{0} \rightarrow$ $\mathrm{SL}_{n}(\mathbb{Z})$ be an injective homomorphism. Then $\phi$ decomposes as $\phi=\epsilon \circ \chi_{h} \circ(-T)^{i}$ where:

○ $\chi_{h}(g)=h^{-1} g h$ is conjugation by some $h \in \mathrm{GL}_{n}(\mathbb{Q})$,

$\circ-T$ is the inverse-transpose map and $i=0$ or 1 , and

$\circ \epsilon$ is an automorphism of $\mathrm{SL}_{n}(\mathbb{Z})$ satisfying $\epsilon(\gamma)= \pm \gamma$.

Proof of Lemma 2.5. We first suppose that $n$ is odd, so $\mathrm{SL}_{n}(\mathbb{R})$ is a simple group. By [Bro89, Theorem IX.5.8], the Zariski closure $H$ of $\phi\left(\Gamma_{0}\right)$ in $\mathrm{SL}_{n}(\mathbb{R})$ is semisimple. Let $\pi_{i}: H \rightarrow H_{i}$ denote the projections of $H$ onto its simple factors. Then $\phi_{i}\left(\Gamma_{0}\right)$ is Zariski dense in $H_{i}$, and so by the Mostow-Margulis superrigidity theorem (see Zim84, Theorem 5.1.2]), $\phi_{i}:=\pi_{i} \circ \phi$ extends to a homomorphism $\Phi_{i}: \mathrm{SL}_{n}(\mathbb{R}) \rightarrow H_{i}$ for each $i$. Since $\mathrm{SL}_{n}(\mathbb{R})$ is simple, there is exactly one $\Phi_{i}$ with infinite image, and it follows that the corresponding factor $H_{i}$ is actually $\mathrm{SL}_{n}(\mathbb{R})$ itself. Hence we have that $\phi$ lifts to an automorphism $\Phi$ of $\mathrm{SL}_{n}(\mathbb{R})$.

Now, it is well known that the outer automorphism group of $\mathrm{SL}_{n}(\mathbb{R})$ is comprised just of the identity and the involution $-T$. In other words, we may decompose $\Phi$ as $\chi_{h} \circ(-T)^{i}$, where $\chi_{h}$ is conjugation by an element $h$ of $\mathrm{SL}_{n}(\mathbb{R})$ and $i=0$ or 1 . (When $n$ is even, it may be necessary to also include conjugation by a permutation matrix $r$ of determinant -1.) Since $\Phi\left(\Gamma_{0}\right)$ is again a lattice of $\mathrm{SL}_{n}(\mathbb{R})$, we clearly have that $h$ commensurates $\mathrm{SL}_{n}(\mathbb{Z})$. By the proof of [Zim84, Proposition 6.2.2] (the statement itself should be slightly modified to accommodate this case), there exists $a \in \mathbb{R}^{*}$ such that $a h \in \mathrm{GL}_{n}(\mathbb{Q})$. Of course, $\chi_{a h}=\chi_{h}$, and so the proof is complete in this case. 
In the case that $n$ is even, $\phi$ determines an embedding $\bar{\phi}: \Gamma_{0} / Z\left(\Gamma_{0}\right) \rightarrow \operatorname{PSL}_{n}(\mathbb{Z})$. One may then carry out the above argument inside $\operatorname{PSL}_{n}(\mathbb{R})$ to obtain that $\bar{\phi}=$ $\chi_{h} \circ(-T)^{i}$. This map lifts to an automorphism $\phi^{\prime}$ of $\mathrm{SL}_{n}(\mathbb{Z})$, and it is immediate that $\phi=\epsilon \circ \phi^{\prime}$, where $\epsilon$ is as required.

In the next proposition we shall use the following notation. For $V \in \operatorname{Gr}_{k}\left(\mathbb{Q}_{p}^{n}\right)$, let $\operatorname{stab}(V)$ denote the stabilizer in $\mathrm{GL}_{n}\left(\mathbb{Q}_{p}\right)$ of $V$, and if $H \leq \mathrm{GL}_{n}\left(\mathbb{Q}_{p}\right)$, then let $\operatorname{stab}_{H}(V)$ denote the stabilizer in $H$ of $V$.

2.6. Proposition. Let $n \geq 3$, and let $V \in \operatorname{Gr}_{k}\left(\mathbb{Q}_{p}^{n}\right)$ and $W \in \operatorname{Gr}_{l}\left(\mathbb{Q}_{p}^{n}\right)$. Suppose that $K \leq \mathrm{SL}_{n}\left(\mathbb{Z}_{p}\right)$ is a subgroup of finite index. If $\operatorname{stab}_{K}(V) \subset \operatorname{stab}_{K}(W)$, then $l=k$ and $W=V$.

Proof. Since $K$ is Zariski dense in $H=\mathrm{SL}_{n}\left(\mathbb{Q}_{p}\right)$ (it is an open subgroup), we have that $\operatorname{stab}_{H}(V) \subset \operatorname{stab}_{H}(W)$. It is well known that $H$ acts primitively on each $k$-Grassmann space, i.e., $H$ acts transitively on $\operatorname{Gr}_{k}\left(\mathbb{Q}_{p}^{n}\right)$ and the stabilizer in $H$ of each point of $\operatorname{Gr}_{k}\left(\mathbb{Q}_{p}^{n}\right)$ is a maximal subgroup of $H$. It follows immediately that we have $\operatorname{stab}_{H}(V)=\operatorname{stab}_{H}(W)$. Now, it is not hard to see that $V$ is uniquely determined by $\operatorname{stab}_{H}(V)$ and so $V=W$.

Proof of Theorem 2.4. In the notation of Lemma 2.5, we have that $\phi=\epsilon \circ \chi_{h} \circ$ $(-T)^{i}$. Since the center of $\mathrm{SL}_{n}(\mathbb{Z})$ acts trivially on $\operatorname{Gr}_{l}\left(\mathbb{Q}_{p}^{n}\right)$, we may suppose that $\epsilon$ is the identity map and that $\phi=\chi_{h} \circ(-T)^{i}$. Having done so, $\phi$ clearly lifts to an automorphism $\Phi$ of $\mathrm{GL}_{n}\left(\mathbb{Q}_{p}\right)$, again defined by the formula $\chi_{h} \circ(-T)^{i}$. For $i=0,1$, let $K_{i}$ denote the closure in $\mathrm{SL}_{n}\left(\mathbb{Z}_{p}\right)$ of $\Gamma_{i}$, so that $X_{i}$ is a homogeneous $K_{i}$-space. Since $\Phi\left(K_{0}\right)$ is a compact group containing $\Gamma_{1}$, we have that $\Phi\left(K_{0}\right) \supset K_{1}$. By the same reasoning, we have $\Phi^{-1}\left(K_{1}\right) \supset K_{0}$, and so $\Phi\left(K_{0}\right)=K_{1}$. Hence, we may define a function $\beta: K_{0} \rightarrow X_{1}$ by

$$
\beta(k)=\Phi(k)^{-1} f\left(k L_{0}\right) .
$$

Then $\beta$ is $\Gamma_{0}$-invariant, since for $\gamma \in \Gamma_{0}$ we have

$$
\beta(\gamma k)=\Phi(\gamma k)^{-1} f\left(\gamma k L_{0}\right)=\Phi(k)^{-1} \Phi(\gamma)^{-1} \phi(\gamma) f\left(k L_{0}\right)=\Phi(k)^{-1} f\left(k L_{0}\right)=\beta(k) .
$$

Hence, by ergodicity of $\Gamma_{0} \curvearrowright K_{0}$, there exists $t \in K_{1}$ such that $\beta(k)=t L_{1}$ for almost all $k \in K_{0}$. It follows that there exists a conull subset $K_{0}^{*} \subset K_{0}$ such that for all $k_{0} \in K_{0}^{*}$,

$$
f\left(k_{0} L_{0}\right)=\Phi\left(k_{0}\right) t L_{1} .
$$

We next argue that this implies that for $k_{0} \in K_{0}^{*}$, if $x=k_{0} L_{0}$, then $\Phi\left(\operatorname{stab}_{K_{0}}(x)\right) \subset$ $\operatorname{stab}_{K_{1}}(f(x))$. Indeed, suppose that $s \in K_{0}$ satisfies $s x=x$. Then choose an element $k \in K_{0}$ such that $k k_{0}, k s k_{0} \in K_{0}^{*}$. (This is possible: since $K_{0}$ is compact it is unimodular, so $K_{0}^{*} k_{0}^{-1}$ and $K_{0}^{*}\left(s k_{0}\right)^{-1}$ are both conull.) It follows that

$$
f(k x)=f\left(k k_{0} L_{0}\right)=\Phi\left(k k_{0}\right) t L_{1}=\Phi(k) \Phi\left(k_{0}\right) t L_{1}=\Phi(k) f(x)
$$

and

$f(k x)=f(k s x)=\Phi\left(k s k_{0} L_{0}\right)=\Phi\left(k s k_{0}\right) t L_{1}=\Phi(k) \Phi(s) \Phi\left(k_{0}\right) t L_{1}=\Phi(k) \Phi(s) f(x)$ and hence $\Phi(s) \in \operatorname{stab}_{K_{1}}(f(x))$.

Finally, since $\Phi$ is either $\chi_{h}$ or $\chi_{h} \circ(-T)$, we have that either $\operatorname{stab}_{K_{1}}\left(h^{-1} x\right) \subset$ $\operatorname{stab}_{K_{1}}(f(x))$ or $\operatorname{stab}_{K_{1}}\left(h^{-1} x^{\perp}\right) \subset \operatorname{stab}_{K_{1}}(f(x))$. In the first case, we can apply Proposition 2.6 to conclude that $l=k$ and $f(x)=h^{-1} x$. In the second case we conclude that $l=n-k$ and $f(x)=h^{-1} x^{\perp}$. 


\section{A SUPERRIGIDITY THEOREM}

In this section, we describe a recent cocycle superrigidity theorem of Adrian Ioana (see [oa07b, Chapter 3] or Ioa07a]). We then derive a corollary which will be used in the proofs of our main theorems. Familiarity with Borel cocycles is needed only to understand the statement of Ioana's theorem and the proof of the corollary. While it may also be possible to use Zimmer's cocycle superrigidity theorem in our arguments, doing so would require a more technical approach.

For $i \in \mathbb{N}$, let $\Gamma \curvearrowright\left(X_{i}, \mu_{i}\right)$ and $\rho_{i}: X_{i+1} \rightarrow X_{i}$ be a factor map (i.e., a $\Gamma$-invariant measure-preserving map). Then the corresponding inverse limit is a $\Gamma$-space $(X, \mu)$ together with factor maps $\pi_{i}: X \rightarrow X_{i}$ satisfying $\pi_{i}=\rho_{i} \circ \pi_{i+1}$ and the usual universal property associated with inverse limits. (The universal property says roughly that any other such $\left(X^{\prime}, \mu^{\prime}\right)$ and $\pi_{i}^{\prime}$ must factor through $(X, \mu)$.)

3.1. Definition. If $\left(X_{i}, \mu_{i}\right)$ are finite $\Gamma$-spaces and $\rho_{i}$ are factor maps as above, then the inverse limit $(X, \mu)$ is called a profinite $\Gamma$-space.

For instance, $\mathrm{SL}_{n}\left(\mathbb{Z}_{p}\right)$ is a profinite $\mathrm{SL}_{n}(\mathbb{Z})$-space, being the inverse limit of the finite $\mathrm{SL}_{n}(\mathbb{Z})$-spaces $\mathrm{SL}_{n}\left(\mathbb{Z} / p^{n} \mathbb{Z}\right)$. Also, if $X_{m}$ denotes the set of $m^{\text {th }}$ congruence components of $\operatorname{Gr}_{k}\left(\mathbb{Q}_{p}^{n}\right)$ described in Section 2, then $\operatorname{Gr}_{k}\left(\mathbb{Q}_{p}^{n}\right)$ will be the inverse limit of the $X_{m}$.

3.2. Theorem ([oa07a, Theorem B]). Suppose that $\Gamma$ is a countable Kazhdan group, and let $(X, \mu)$ be a profinite $\Gamma$-space with corresponding factor maps $\pi_{i}: X \rightarrow$ $X_{i}$. Suppose additionally that the action $\Gamma \curvearrowright(X, \mu)$ is ergodic and free. If $\alpha: \Gamma \times$ $X \rightarrow \Lambda$ is a cocycle into an arbitrary countable group $\Lambda$, then there exists $i \in \mathbb{N}$ such that $\alpha$ is cohomologous to a cocycle $\Gamma \times X_{i} \rightarrow \Lambda$. More precisely, there exists $i \in \mathbb{N}$ and a cocycle $\alpha_{i}: \Gamma \times X_{i} \rightarrow \Lambda$ such that $\alpha$ is cohomologous to the cocycle $\alpha^{\prime}$ defined by $\alpha^{\prime}(g, x)=\alpha_{i}\left(g, \pi_{i}(x)\right)$.

This is most useful in the case when $\alpha$ is a cocycle corresponding to a Borel homomorphism $f$ from $E_{\Gamma}$ to $E_{\Lambda}$, where $E_{\Lambda}$ is some orbit equivalence relation. In this case, the theorem gives hypotheses under which $f$ can be replaced with an action-preserving map.

3.3. Corollary ([Ioa07a, Theorem 4.1]). Suppose that $\Gamma$ is a countable Kazhdan group, and let $(X, \mu)$ be a free, ergodic profinite $\Gamma$-space. Let $\Lambda$ be a countable group and let $\Lambda \curvearrowright Y$ be a free action. Suppose that $f$ is a Borel homomorphism from $E_{\Gamma}$ to $E_{\Lambda}$. Then there exists an ergodic component $\Gamma_{0} \curvearrowright X_{0}$ for $\Gamma \curvearrowright X$ and a permutation group homomorphism $\left(\phi, f^{\prime}\right): \Gamma_{0} \curvearrowright X_{0} \longrightarrow \Lambda \curvearrowright Y$ such that for all $x \in X_{0}$, we have that $f^{\prime}(x) E_{\Lambda} f(x)$.

Proof. Let $\alpha: \Gamma \curvearrowright X \rightarrow \Lambda$ be the cocycle corresponding to $f$. By Theorem 3.2, there exists a finite factor $\left(X^{\prime}, \mu^{\prime}\right)$ of $(X, \mu)$ (denote the projection map by $\pi$ ), and a Borel function $b: X \rightarrow \Lambda$ such that the adjusted cocycle

$$
\alpha^{\prime}(g, x):=b(g x) \alpha(g, x) b(x)^{-1}
$$

depends only on $g$ and $\pi(x)$. Choose any $x_{0} \in X^{\prime}$, and let $\Gamma_{0}$ be the stabilizer of $x_{0}$ in $\Gamma$. Clearly $\Gamma_{0} \leq \Gamma$ is a subgroup of finite index, and by [oa07a, Remark 1.3(3)], $X_{0}:=\pi^{-1}\left(x_{0}\right)$ is an ergodic component for the action of $\Gamma_{0}$. Since $\pi$ is constant on $X_{0}$, the restriction of $\alpha^{\prime}$ to $\Gamma_{0} \times X_{0}$ is independent of $x \in X_{0}$. It follows that $\phi(\gamma):=\alpha^{\prime}(\gamma, \cdot)$ defines a homomorphism $\Gamma_{0} \rightarrow \Lambda$, and then letting $f^{\prime}=b f$ it is 
easily seen that $\left(\phi, f^{\prime}\right)$ satisfies our requirements. (Of course, since $\alpha^{\prime}$ need only satisfy the cocycle identity almost everywhere, one may need to delete a null set of $X_{0}$.)

We first use this corollary to establish the following Borel incomparability result.

3.4. Definition. Fix $n \in \mathbb{N}$ and $p$ prime. Let $E_{\mathrm{SL}_{n} \mathbb{Z}}^{k}$ and $E_{\mathrm{GL}_{n} \mathbb{Q}}^{k}$ denote the orbit equivalence relations induced on $\mathrm{Gr}_{k}\left(\mathbb{Q}_{p}^{n}\right)$ by $\mathrm{SL}_{n}(\mathbb{Z})$ and $\mathrm{GL}_{n}(\mathbb{Q})$, respectively.

3.5. Theorem. Suppose that $k, l<n$ and $l$ is neither $k$ nor $n-k$. Then $E_{\mathrm{GL}_{n} \mathbb{Q}}^{k}$ is Borel incomparable with $E_{\mathrm{GL}_{n} \mathbb{Q}}^{l}$.

In the next section, we shall see that this theorem implies that the quasiisomorphism relations on $R(n, p, k), R(n, p, l)$ are Borel incomparable. We prove slightly more:

3.6. Theorem. Let $n \geq 4$ and $k, l<n$, and suppose that $l$ is neither $k$ nor $n-k$.

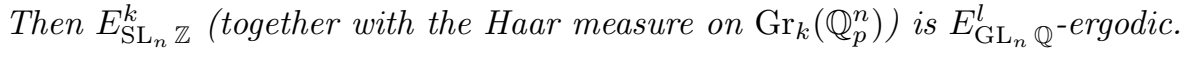

Theorem 3.5 follows immediately; any Borel reduction from $E_{\mathrm{GL}_{n} \mathbb{Q}}^{k}$ to $E_{\mathrm{GL}_{n} \mathbb{Q}}^{l}$ is clearly a weak Borel reduction from $E_{\mathrm{SL}_{n} \mathbb{Z}}^{k}$ to $E_{\mathrm{GL}_{n} \mathbb{Q}}^{l}$, and Theorem 3.6 implies that $E_{\mathrm{SL}_{n} \mathbb{Z}}^{k} \mathbb{Z}_{B}^{w} E_{\mathrm{GL}_{n} \mathbb{Q}}^{l}$.

Proof of Theorem 3.6. Let $f: \operatorname{Gr}_{k}\left(\mathbb{Q}_{p}^{n}\right) \rightarrow \operatorname{Gr}_{l}\left(\mathbb{Q}_{p}^{n}\right)$ be a Borel homomorphism from $E_{\mathrm{SL}_{n} \mathbb{Z}}^{k}$ to $E_{\mathrm{GL}_{n} \mathbb{Q}}^{l}$, and suppose towards a contradiction that $f$ does not map a conull set into a single $\mathrm{GL}_{n}(\mathbb{Q})$-orbit. We first reduce the analysis to a situation where the hypotheses of Corollary 3.3 hold. Since $\operatorname{SL}_{n}(\mathbb{Z})$ acts ergodically on $\operatorname{Gr}_{k}\left(\mathbb{Q}_{p}^{n}\right)$, we need only argue that both $\mathrm{SL}_{n}(\mathbb{Z}) \curvearrowright \mathrm{Gr}_{k}\left(\mathbb{Q}_{p}^{n}\right)$ and $\mathrm{GL}_{n}(\mathbb{Q}) \curvearrowright \mathrm{Gr}_{l}\left(\mathbb{Q}_{p}^{n}\right)$ are free actions. While neither action is literally free, it will suffice to establish the following statements:

3.7. Claim (a). The action of $\operatorname{PSL}_{n}(\mathbb{Z})$ on $\operatorname{Gr}_{k}\left(\mathbb{Q}_{p}^{n}\right)$ is almost free.

3.8. Claim (b). The function $f$ maps a (Haar) conull set into the free part of the action of $\mathrm{PGL}_{n}(\mathbb{Q})$ on $\mathrm{Gr}_{l}\left(\mathbb{Q}_{p}^{n}\right)$.

Here, if the countable group $\Gamma$ acts on a standard Borel space $X$, then we let

$$
\operatorname{Fr}(\Gamma \curvearrowright X):=\{x \in X: 1 \neq g \in \Gamma \Longrightarrow g x \neq x\}
$$

denote the free part of the action of $\Gamma$ on $X$. If $X$ carries a (not necessarily $\Gamma$-invariant) probability measure, then we say that $\Gamma \curvearrowright X$ is almost free iff $\operatorname{Fr}(\Gamma \curvearrowright X)$ is conull.

3.9. Lemma (essentially [Tho02, Lemma 5.1]). Suppose that $f: \operatorname{Gr}_{k}\left(\mathbb{Q}_{p}^{n}\right) \rightarrow \operatorname{Gr}_{l}\left(\mathbb{Q}_{p}^{n}\right)$ is a Borel homomorphism from $E_{\mathrm{SL}_{n} \mathbb{Z}}^{k}$ to $E_{\mathrm{GL}_{n} \mathbb{Q}}^{l}$. Then either $f$ maps a conull set into a single $\mathrm{GL}_{n}(\mathbb{Q})$-orbit, or there exists a conull $X \subset \mathrm{Gr}_{k}\left(\mathbb{Q}_{p}^{n}\right)$ such that

$$
f(X) \subset \operatorname{Fr}\left(\mathrm{PGL}_{n}(\mathbb{Q}) \curvearrowright \mathrm{Gr}_{l}\left(\mathbb{Q}_{p}^{n}\right)\right) .
$$

It is clear that the lemma establishes Claim (b); Claim (a) also follows by applying it in the case when $l=k$ and $f$ is the identity map on $\operatorname{Gr}_{k}\left(\mathbb{Q}_{p}^{n}\right)$. (While Thomas only stated Lemma 3.9 for the special case when $k=n-1$, his argument goes through without change for arbitrary $k, l$.) 
3.10. Claim. We may suppose that there exists an ergodic component $\Gamma_{0} \curvearrowright X_{0}$ for the action $\mathrm{SL}_{n}(\mathbb{Z}) \curvearrowright \mathrm{Gr}_{k}\left(\mathbb{Q}_{p}^{n}\right)$ and a homomorphism $\phi: \Gamma_{0} \rightarrow \mathrm{GL}_{n}(\mathbb{Q})$ such that

$$
(\phi, f): \Gamma_{0} \curvearrowright X_{0} \longrightarrow \mathrm{GL}_{n}(\mathbb{Q}) \curvearrowright \mathrm{Gr}_{l}\left(\mathbb{Q}_{p}^{n}\right)
$$

is a homomorphism of permutation groups.

In the proof, we will in fact produce a $\phi$ such that $\phi\left(\Gamma_{0}\right)$ is a subgroup of $\mathrm{SL}_{n}(\mathbb{Z})$ of finite index.

Proof of claim. Using Claims (a) and (b) together, it is not difficult to see that we may apply Corollary 3.3 to suppose that there exists an ergodic component $\bar{\Gamma}_{0} \curvearrowright X_{0}$ for $\operatorname{PSL}_{n}(\mathbb{Z}) \curvearrowright \operatorname{Gr}_{k}\left(\mathbb{Q}_{p}^{n}\right)$ and a homomorphism of permutation groups

$$
(\bar{\phi}, f): \bar{\Gamma}_{0} \curvearrowright X_{0} \longrightarrow \mathrm{PGL}_{n}(\mathbb{Q}) \curvearrowright \operatorname{Gr}_{l}\left(\mathbb{Q}_{p}^{n}\right) .
$$

We wish to lift $\phi$ to a map $\Gamma_{0} \rightarrow \mathrm{GL}_{n}(\mathbb{Q})$, where $\Gamma_{0}$ is the preimage in $\mathrm{SL}_{n}(\mathbb{Z})$ of $\bar{\Gamma}_{0}$.

First, suppose that $\bar{\phi}$ is not injective. In this case, by Margulis's theorem on normal subgroups [Zim84, Theorem 8.1.2], the kernel of $\bar{\phi}$ has finite index in $\bar{\Gamma}_{0}$. Hence, $\bar{\phi}$ has finite image and so, passing to an ergodic subcomponent, we can suppose without loss of generality that $\bar{\phi}=1$. This implies that $f$ is $\bar{\Gamma}_{0}$-invariant and since $\bar{\Gamma}_{0} \curvearrowright X_{0}$ is ergodic, $f$ is almost constant. Hence, in this case, $f$ maps a conull set into a single $\mathrm{GL}_{n}(\mathbb{Q})$-orbit, which is a contradiction.

Next, suppose that $\bar{\phi}$ is injective. In this case, we shall again make use of Margulis's results. The next lemma will be used in tandem with Lemma 2.5 ,

3.11. Lemma. If $\Gamma_{0} \leq \mathrm{SL}_{n}(\mathbb{Z})$ is a finite index subgroup and $\phi: \Gamma_{0} \rightarrow \mathrm{GL}_{n}(\mathbb{Q})$ is a homomorphism, then there exists a finite index subgroup $\Lambda \leq \Gamma_{0}$ such that $\phi(\Lambda) \leq \mathrm{SL}_{n}(\mathbb{Z})$ is a subgroup of finite index.

Proof. Since $\Gamma_{0}$ is Kazhdan (see Lub03, Theorem 1.5]), we have that $\Gamma_{0}^{\prime}:=\left[\Gamma_{0}, \Gamma_{0}\right]$ is a finite index subgroup of $\Gamma_{0}$ (see Lub03, Corollary 1.29]). Now since $\mathrm{GL}_{n}(\mathbb{Q}) / \mathrm{SL}_{n}(\mathbb{Q}) \cong \mathbb{Q}^{\times}$is abelian, we have that

$$
\phi\left(\Gamma_{0}^{\prime}\right) \leq\left[\mathrm{GL}_{n}(\mathbb{Q}), \mathrm{GL}_{n}(\mathbb{Q})\right] \leq \mathrm{SL}_{n}(\mathbb{Q}) .
$$

(In fact, the latter $\leq$ is an equality.) Hence, replacing $\Gamma_{0}$ by $\Gamma_{0}^{\prime}$ if necessary, we may suppose without loss of generality that $\phi\left(\Gamma_{0}\right) \subset \mathrm{SL}_{n}(\mathbb{Q})$. Repeating the proof of Lemma 2.5. we may suppose that $\phi$ extends to an automorphism of $\mathrm{SL}_{n}(\mathbb{R})$. (Once again, in the case that $n$ is even, this argument should be carried out inside $\mathrm{PSL}_{n}(\mathbb{R})$. Hence one may need to replace $\phi$ by $\epsilon \circ \phi$, where $\epsilon$ is as in the notation of Lemma 2.5.) It follows that $\phi\left(\Gamma_{0}\right)$ is again a lattice of $\mathrm{SL}_{n}(\mathbb{R})$. Since $\phi\left(\Gamma_{0}\right) \subset$ $\mathrm{SL}_{n}(\mathbb{Q})$, by $\left[\right.$ Bro89, IX.4.14] we have that $\phi\left(\Gamma_{0}\right)$ is commensurable with $\mathrm{SL}_{n}(\mathbb{Z})$, and the lemma follows.

Although Lemma 3.11 has been stated so that it will be useful later on, for the present circumstances let us note that the same proof easily applies to the case of homomorphisms into $\mathrm{PGL}_{n}(\mathbb{Q})$. In other words, replacing $X_{0}$ with a smaller ergodic component, we may suppose without loss of generality that $\bar{\phi}\left(\bar{\Gamma}_{0}\right)$ is a subgroup of $\mathrm{PSL}_{n}(\mathbb{Z})$ of finite index. Moreover, the proof of Lemma 2.5 shows that $\bar{\phi}$ lifts to a homomorphism $\phi: \Gamma_{0} \rightarrow \mathrm{SL}_{n}(\mathbb{Z})$. Since $\phi$ is a lifting, we easily obtain that $f(\gamma x)=\phi(\gamma) f(x)$ for all $\gamma \in \Gamma_{0}$, which completes the proof of the claim. 
We now wish to maneuver into a situation where we can apply Theorem 2.4.

3.12. Claim. We may suppose that $f\left(X_{0}\right) \subset X_{1}$, where $X_{1}$ is an ergodic component for the action of $\phi\left(\Gamma_{0}\right)$ on $\operatorname{Gr}_{l}\left(\mathbb{Q}_{p}^{n}\right)$.

Proof of claim. Let $Z_{1}, \ldots, Z_{m}$ be the ergodic components for the action of $\phi\left(\Gamma_{0}\right)$ on $\operatorname{Gr}_{l}\left(\mathbb{Q}_{p}^{n}\right)$. Now, each $f^{-1}\left(Z_{i}\right)$ is $\Gamma_{0}$-invariant, and since $\Gamma_{0} \curvearrowright X_{0}$ is ergodic, exactly one of the $f^{-1}\left(Z_{i}\right)$ is conull. Deleting a null subset of $X_{0}$, we may suppose that $f\left(X_{0}\right) \subset Z_{i}$, as desired.

Finally, we may apply Theorem 2.4 to conclude that $l=k$ or $l=n-k$, contradicting our initial hypothesis. This completes the proof of Theorem 3.6 .

\section{Completion of local torsion-Free abelian groups}

In this section, we recall some facts surrounding the Kurosh-Malcev completion of a $p$-local torsion-free abelian group. The completion map will allow us to relate the space of $p$-local torsion-free abelian groups of a fixed divisible rank with the $k$-Grassmann space $\operatorname{Gr}_{k}\left(\mathbb{Q}_{p}^{n}\right)$. Using this idea, Thomas essentially showed [Tho03a, Theorem 4.7] that the quasi-isomorphism relation $\sim_{n, p}^{k}$ on $R(n, p, k)$ is bireducible with the orbit equivalence relation $E_{\mathrm{GL}_{n} \mathbb{Q}}^{k}$ induced by the action of $\mathrm{GL}_{n}(\mathbb{Q})$ or $\operatorname{Gr}_{k}\left(\mathbb{Q}_{p}^{n}\right)$. (Together with Theorem 3.5, this implies that $\sim_{n, p}^{k}$ is Borel incomparable with $\sim_{n, p}^{l}$ for $l \neq k, n-k$.) We shall use the completion to establish the following more technical result.

4.1. Lemma. There exists an equivalence relation $E_{\cong}^{k}$ on the space $\operatorname{Gr}_{k}\left(\mathbb{Q}_{p}^{n}\right)$ satisfying:

(a) $E_{\mathrm{SL}_{n} \mathbb{Z}}^{k} \subset E_{\cong}^{k} \subset E_{\mathrm{GL}_{n} \mathbb{Q}}^{k}$, and

(b) $E_{\cong}^{k}$ is Borel bireducible with the isomorphism relation $\cong_{n, p}^{k}$ on $R(n, p, k)$.

Granting this lemma, we can prove the following:

4.2. Theorem (Theorem A, case 1 ). Let $n \geq 4$ and $k, l<n$, and suppose that $l$ is neither $k$ nor $n-k$. Then $\cong_{n, p}^{k}$ is Borel incomparable with $\cong_{n, p}^{l}$.

Proof. By part (b) of Lemma 4.1, it suffices to prove that the relations $E_{\cong}^{k}$ and $E_{\cong}^{l}$ are Borel incomparable. Now, if $f$ is a Borel reduction from $E_{\cong}^{k}$ to $E_{\cong}^{l}$, then using part (a) of Lemma 4.1 we clearly have that $f$ is a weak Borel reduction from $E_{\mathrm{SL}_{n} \mathbb{Z}}^{k}$

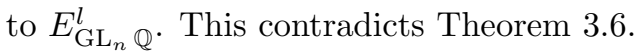

The completion map. Following Fuc73, for $A \in R(n, p)$ we define the completion of $A$ to a $\mathbb{Z}_{p}$-submodule of $\mathbb{Q}_{p}^{n}$ by

$$
\Lambda(A):=A \otimes \mathbb{Z}_{p}
$$

In other words, $\Lambda(A)$ is just the set of all $\mathbb{Z}_{p}$-linear combinations of elements of $A$, where $A$ is considered as a subset of $\mathbb{Q}_{p}^{n}$. The completion map takes values in the standard Borel space $\mathcal{M}(n, p)$ of $\mathbb{Z}_{p}$-submodules of $\mathbb{Q}_{p}^{n}$ with $\mathbb{Z}_{p}$-rank exactly equal to $n$. In fact, by Fuc73, 93.1,93.5], $\Lambda$ is a bijection between $R(n, p)$ and $\mathcal{M}(n, p)$.

Now, if $M \in \mathcal{M}(n, p)$, then by [Fuc73, 93.3], $M$ can be decomposed as a direct sum

$$
M=V_{M} \oplus L
$$


where $V_{M}$ is a vector subspace of $\mathbb{Q}_{p}^{n}$ and $L$ is a free $\mathbb{Z}_{p}$-submodule of $\mathbb{Q}_{p}^{n}$. The vector subspace $V_{M}$ is uniquely determined by $M$. There are many possible complementary submodules $L$, but in any case we have $\operatorname{rank} L=n-\operatorname{dim} V$.

By [Fuc73, Exercise 93.1], the dimension of $V_{\Lambda(A)}$ is precisely the divisible rank of $A$. Letting $\mathcal{M}(n, p, k)$ denote the subspace of $\mathcal{M}(n, p)$ consisting of just those $M$ with $\operatorname{dim} V_{M}=k$, it follows that $\Lambda$ is a bijection of $R(n, p, k)$ with $\mathcal{M}(n, p, k)$. Since $\Lambda$ is $\mathrm{GL}_{n}(\mathbb{Q})$-invariant, we have that $\cong_{n, p}^{k}$ is Borel equivalent (i.e., isomorphic via a Borel map) to the orbit equivalence relation induced by the action of $\mathrm{GL}_{n}(\mathbb{Q})$ on $\mathcal{M}(n, p, k)$.

Decomposition of the space of completed groups. We now investigate the fibers of the "vector space part" map from $\mathcal{M}(n, p, k)$ to $\operatorname{Gr}_{k}\left(\mathbb{Q}_{p}^{n}\right)$ defined by $M \mapsto$ $V_{M}$, where $V_{M}$ is as in (4.3). For this purpose, fix $V \in \operatorname{Gr}_{k}\left(\mathbb{Q}_{p}^{n}\right)$ and let $M \in$ $\mathcal{M}(n, p, k)$ be an arbitrary module such that $V_{M}=V$. If $W$ is any complementary subspace of $V$, meaning that $V \cap W=0$ and $V \oplus W=\mathbb{Q}_{p}^{n}$, then $M$ can always be written (uniquely) as $V \oplus L$, where $L \leq W$. Since $L$ is free and $\operatorname{rank} L=\operatorname{dim} W$, the set $\left\{M \in \mathcal{M}(n, p, k): V_{M}=V\right\}$ is in bijection with the lattices of $W$.

4.4. Definition. Let $K$ be a discrete valuation field and $R$ its ring of integers. Then a lattice of $K^{l}$ is a free $R$-submodule of $K^{l}$ of rank $l$. Equivalently, a lattice of $K^{l}$ is the $R$-span of $l$ linearly independent elements of $K^{l}$. We denote the set of lattices of $K^{l}$ by $\mathcal{L}\left(K^{l}\right)$.

By the discussion in $\left[\right.$ Ser03, Section II.1.1], since $\mathbb{Q}$ is a dense subfield of $\mathbb{Q}_{p}$, the map $L \mapsto \mathbb{Z}_{p} \otimes L$ is a bijection between the lattices of $\mathbb{Q}^{l}$ (with respect to the $p$-adic valuation on $\mathbb{Q}^{*}$ ) and the lattices of $\mathbb{Q}_{p}^{l}$. Hence, any lattice of $\mathbb{Q}_{p}^{l}$ may be expressed as the $\mathbb{Z}_{p}$-span of $l$ linearly independent elements of $\mathbb{Q}^{l}$. In particular, there are only countably many lattices of $\mathbb{Q}_{p}^{l}$ and so the vector space part map $M \mapsto V_{M}$ is countable-to-one.

Since any countable-to-one Borel function between standard Borel spaces admits a Borel section, there clearly exists a Borel bijection $f: \operatorname{Gr}_{k}\left(\mathbb{Q}_{p}^{n}\right) \times \mathcal{L}\left(\mathbb{Q}_{p}^{n-k}\right) \rightarrow$ $\mathcal{M}(n, p, k)$ satisfying $V_{f(V, L)}=V$ for all $V, L$. It will be useful to work with a particular such function.

4.5. Definition. If $V \in \operatorname{Gr}_{k}\left(\mathbb{Q}_{p}^{n}\right)$, let $V^{c}$ be the unique complementary subspace of $V$ spanned by the basis vectors $e_{j_{1}}, \ldots, e_{j_{n-k}}$ with the following properties:

(a) The $\mathbb{Z}_{p}$-span of $\left(V \cap \mathbb{Z}_{p}^{n}\right) \cup\left(\mathbb{Z}_{p} e_{j_{1}} \oplus \cdots \oplus \mathbb{Z}_{p} e_{j_{n-k}}\right)$ is all of $\mathbb{Z}_{p}^{n}$, and

(b) $\left\langle j_{i}\right\rangle$ is the lexicographically greatest sequence satisfying (a).

We call $V^{c}$ the canonical complementary subspace of $V$.

We now discuss how to find and identify such a sequence $\left\langle j_{i}\right\rangle$; in particular, we will show that $V^{c}$ exists. The key is that we can write $V$ as the column space of an $n \times k$ matrix $A$ satisfying:

- Each row of the $k \times k$ identity matrix appears as a row of $A$ (call these the pivot rows), and

○ every entry of $A$ is in $\mathbb{Z}_{p}$.

(To obtain such a matrix, begin with an arbitrary matrix whose column space is $V$. Rescale the first column so that all entries are $p$-adic integers and at least one entry is 1 . Use this 1 to zero out the other entries in its row. Repeat for the second column, etc.) It is easily seen that the sequence $j_{i}$ of indices of the nonpivot rows 
of $A$ satisfies (a). Our requirement that $\left\langle j_{i}\right\rangle$ is lex-greatest amounts to the more natural assertion that the sequence of indices of the pivot rows of $A$ is lex-least.

For example, we have already seen in (2.2) that any $V \in\left(K_{p^{t}}\right) V_{0}$ can be written as the column space of a matrix of the form $\left[\begin{array}{c}I_{k} \\ v\end{array}\right]$, where the entries of $v$ are in $\mathbb{Z}_{p}$. It follows that $V$ has the canonical complementary subspace $V^{c}=\mathbb{Q}_{p} e_{k+1} \oplus \cdots \oplus \mathbb{Q}_{p} e_{n}$.

Now, for a lattice $L \leq \mathbb{Q}_{p}^{n-k}$, let $L[V]$ be the isomorphic copy of $L$ inside $V^{c}$ induced by following the obvious map (that is, the linear map defined by $e_{i} \mapsto e_{j_{i}}$ ). We define the adjoining of $V$ and $L$ by

$$
(V, L):=V \oplus L[V] .
$$

It is clear from our construction that adjoining defines a Borel bijection

$$
(\cdot, \cdot): \operatorname{Gr}_{k}\left(\mathbb{Q}_{p}^{n}\right) \times \mathcal{L}\left(\mathbb{Q}_{p}^{n-k}\right) \rightarrow \mathcal{M}(n, p, k),
$$

which we shall use to parametrize the elements of $\mathcal{M}(n, p, k)$.

Relations on $k$-Grassmann space. Our approach to Lemma 4.1 will be to investigate the "canonical" copy of $\operatorname{Gr}_{k}\left(\mathbb{Q}_{p}^{n}\right)$ in $\mathcal{M}(n, p, k)$. Letting $L_{0}$ denote the standard lattice $\mathbb{Z}_{p}^{n-k}$ of $\mathbb{Q}_{p}^{n-k}$, we put $Y_{0}:=\left\{\left(V, L_{0}\right): V \in \operatorname{Gr}_{k}\left(\mathbb{Q}_{p}^{n}\right)\right\}$. We first give the following characterization.

4.6. Proposition. $Y_{0}$ is precisely the orbit $\left(\mathrm{SL}_{n} \mathbb{Z}_{p}\right) M_{0}$, where $M_{0}$ is the module

$$
M_{0}=\left(V_{0}, L_{0}\right)=\left(\mathbb{Q}_{p} e_{1} \oplus \cdots \oplus \mathbb{Q}_{p} e_{k}\right) \oplus\left(\mathbb{Z}_{p} e_{k+1} \oplus \cdots \oplus \mathbb{Z}_{p} e_{n}\right) .
$$

Proof. We need only show that for any $g \in \mathrm{SL}_{n}\left(\mathbb{Z}_{p}\right)$, we have $g\left(V_{0}, L_{0}\right) \in Y_{0}$. Then, since $\mathrm{SL}_{n}\left(\mathbb{Z}_{p}\right)$ acts transitively on $\operatorname{Gr}_{k}\left(\mathbb{Q}_{p}^{n}\right)$, it follows that $Y_{0}$ is precisely the orbit $\left(\mathrm{SL}_{n} \mathbb{Z}_{p}\right)\left(V_{0}, L_{0}\right)$.

Suppose first that $g\left(V_{0}, L_{0}\right)$ is of the form $\left(V_{0}, L\right)$; in other words, suppose that $g V_{0}=V_{0}$. In this case, $g$ acts on the quotient $\mathbb{Q}_{p}^{n} / V_{0}$ (in the basis represented by $\left.e_{k+1}, \ldots, e_{n}\right)$ via its $(n-k) \times(n-k)$ lower right-hand corner $g^{c}$. It follows easily that $g\left(V_{0}, L_{0}\right)=\left(V_{0}, g^{c} L_{0}\right)$, and since the entries of $g^{c}$ lie in $\mathbb{Z}_{p}$, we clearly have $g^{c} L_{0}=L_{0}$. Hence, $g\left(V_{0}, L_{0}\right)=\left(V_{0}, L_{0}\right)$ is an element of $Y_{0}$.

Now suppose that $g\left(V_{0}, L_{0}\right)=(V, L)$ is arbitrary. It suffices to show that there exists $g_{1} \in \mathrm{SL}_{n}\left(\mathbb{Z}_{p}\right)$ such that $g_{1}\left(V_{0}, L\right)=(V, L)$, for then, $g_{1}^{-1} g\left(V_{0}, L_{0}\right)=\left(V_{0}, L\right)$ and we are in the previous case. Permuting the standard basis if necessary, we can suppose that $V=\operatorname{col}\left[\begin{array}{c}I_{k} \\ v\end{array}\right]$, where the entries of $v$ are in $\mathbb{Z}_{p}$. It follows easily that $g_{1}:=\left[\begin{array}{cc}I_{k} & 0 \\ v & I_{n-k}\end{array}\right]$ satisfies our requirements.

4.7. Remark. While $Y_{0}$ is not invariant for the action of $\mathrm{GL}_{n}(\mathbb{Q})$, the last proposition shows in particular that $Y_{0}$ is invariant for the action of the subgroup $\mathrm{SL}_{n}\left(\mathbb{Z}_{(p)}\right)$. However, it is not difficult to see that the restriction of the orbit equivalence relation induced by the action of $\mathrm{GL}_{n}(\mathbb{Q})$ on $\mathcal{M}(n, p, k)$ to $Y_{0}$ is not induced by the action of any subgroup of $\mathrm{GL}_{n}(\mathbb{Q})$.

4.8. Proposition. $Y_{0}$ is a complete Borel section for the orbit equivalence relation induced by the action of $\mathrm{GL}_{n}(\mathbb{Q})$ on $\mathcal{M}(n, p, k)$.

Here, a Borel subset $A \subset X$ is said to be a complete Borel section for the equivalence relation $E$ on $X$ iff $A$ meets every $E$-class. A countable Borel equivalence relation is bireducible with its restriction to any complete Borel section. Hence, 
using the obvious bijection of $Y_{0}$ with $\operatorname{Gr}_{k}\left(\mathbb{Q}_{p}^{n}\right)$, we obtain that the orbit equivalence relation induced by the action of $\mathrm{GL}_{n}(\mathbb{Q})$ on $\mathcal{M}(n, p, k)$ is bireducible with the following equivalence relation on $\operatorname{Gr}_{k}\left(\mathbb{Q}_{p}^{n}\right)$ :

4.9. Definition. For $V, V^{\prime} \in \mathrm{Gr}_{k}\left(\mathbb{Q}_{p}^{n}\right)$, we define that $V E_{\cong}^{k} V^{\prime}$ iff there exists $g \in \mathrm{GL}_{n}(\mathbb{Q})$ such that $\left(V, L_{0}\right)=g\left(V^{\prime}, L_{0}\right)$.

Proof of Proposition 4.8. We must prove that for every $(V, L) \in \mathcal{M}(n, p, k)$, there exists $g \in \mathrm{GL}_{n}(\mathbb{Q})$ and $V^{\prime}$ such that $g(V, L)=\left(V^{\prime}, L_{0}\right)$. (Of course, $V^{\prime}$ will be $g V$.) Permuting the standard basis if necessary, we may suppose that $V=\operatorname{col}\left[\begin{array}{c}I_{k} \\ v\end{array}\right]$, where the entries of $v$ are in $\mathbb{Z}_{p}$. Recall that $L$ has a rational basis over $\mathbb{Z}_{p}$, and so there exists $h \in \mathrm{GL}_{n-k}(\mathbb{Q})$ such that $h L=L_{0}$. Now, choose a rational matrix $j$ so that the entries of $j+h v$ are in $\mathbb{Z}_{p}$, and let $g=\left[\begin{array}{cc}I_{k} & 0 \\ j & h\end{array}\right]$. Then both $V$ and $g V=$ $\operatorname{col}\left[\begin{array}{c}I_{k} \\ j+h v\end{array}\right]$ have the canonical complementary subspace $V_{1}=\mathbb{Q}_{p} e_{k+1} \oplus \cdots \oplus \mathbb{Q}_{p} e_{n}$. One now easily computes that $g(V, L)=(g V, h L)=\left(g V, L_{0}\right)$, as desired.

Proof of Lemma 4.1, To see that $E_{\mathrm{SL}_{n} \mathbb{Z}}^{k} \subset E_{\cong}^{k}$, suppose that $g \in \mathrm{SL}_{n}(\mathbb{Z})$ and $g V=V^{\prime}$. Then by Proposition 4.6, we have $g\left(V, L_{0}\right)=\left(V^{\prime}, L_{0}\right)$ and so $V E \cong E^{k}$. To see that $E_{\cong}^{k} \subset E_{\mathrm{GL}_{n}}^{k} \mathbb{Q}$, notice that if $g \in \mathrm{GL}_{n}(\mathbb{Q})$ and $g\left(V, L_{0}\right)=\left(V^{\prime}, L_{0}\right)$, then $g V=V^{\prime}$. We have already remarked that the bireducibility of $E_{\cong}^{k}$ and $\cong_{n, p}^{k}$ follows from Proposition 4.8 .

\section{The PRoOfs of The MAIN THEOREMS}

5.1. Theorem (Theorem B, part 1). If $n \geq 3$ and $k \leq n-2$, then $\cong_{n, p}^{k} \mathbb{\leq}_{B} \sim_{n, p}^{k}$.

By [Tho02, Theorem 4.3], the quasi-isomorphism relation $\sim_{n, p}^{k}$ is bireducible with $E_{\mathrm{GL}_{n} \mathbb{Q}}^{k}$. Using this together with Lemma 4.1, we reduce Theorem 5.1 to the following statement.

5.2. Theorem. If $n \geq 3$ and $k \leq n-2$, then $E_{\cong}^{k} \mathbb{S}_{B} E_{\mathrm{GL}_{n} \mathbb{Q}}^{k}$.

It is worth remarking that this result gives new examples of countable Borel equivalence relations $E \subset F$ such that $E \not_{B} F$ (see [Ada02]). Before the proof, we introduce a key invariant on the space $\mathcal{L}\left(\mathbb{Q}_{p}^{l}\right)$ of lattices of $\mathbb{Q}_{p}^{l}$.

5.3. Definition. For a lattice $L \in \mathcal{L}\left(\mathbb{Q}_{p}^{l}\right)$, let $A$ be any $l \times l$ matrix over $\mathbb{Q}_{p}$ whose columns form a $\mathbb{Z}_{p}$-basis for $L$. The type of $L$, denoted type $(L)$, is the reduction modulo $l$ of $\nu_{p}(\operatorname{det} A)$. (Here, $\nu_{p}$ denotes the $p$-adic valuation on $\mathbb{Q}_{p}^{*}$.)

It is easily checked that the type is independent of the choice of the matrix $A$. Moreover:

5.4. Proposition. If $s \in \mathrm{GL}_{l}\left(\mathbb{Q}_{p}\right)$, then $\operatorname{type}(s L) \equiv \nu_{p}(\operatorname{det} s)+\operatorname{type}(L)$, modulo l.

In particular, the type of a lattice $L$ depends only on its class $\Lambda=\left\{a L: a \in \mathbb{Q}_{p}\right\}$.

5.5. Remark. In the case when $l=2$, there is a natural graph structure on the set of lattice classes. Join $\Lambda$ and $\Lambda^{\prime}$ by an edge iff there are $L \in \Lambda$ and $L^{\prime} \in \Lambda^{\prime}$ such that $L^{\prime}$ is a maximal proper sublattice of $L$ (or vice versa). The resulting graph is the $(p+1)$-regular tree and the types correspond to the colors in a 2-coloring of the tree. See the cover of the most recent printing of [Ser03] for a picture in the case that $l=p=2$. 
Proof of Theorem [5.2. Suppose that $f: \operatorname{Gr}_{k}\left(\mathbb{Q}_{p}^{n}\right) \rightarrow \operatorname{Gr}_{k}\left(\mathbb{Q}_{p}^{n}\right)$ is a Borel reduction from $E_{\cong}^{k}$ to $E_{\mathrm{GL}_{n} \mathbb{Q}}^{k}$. Then clearly $f$ is a weak Borel reduction from $E_{\mathrm{SL}_{n} \mathbb{Z}}^{k}$ to $E_{\mathrm{GL}_{n} \mathbb{Q}}^{k}$. Repeating arguments from the proof of Theorem 3.6. we may suppose there is an ergodic component $\Gamma_{0} \curvearrowright X_{0}$ for $\mathrm{SL}_{n}(\mathbb{Z}) \curvearrowright \operatorname{Gr}_{k}\left(\mathbb{Q}_{p}^{n}\right)$ and a homomorphism $\phi: \Gamma_{0} \rightarrow$ $\mathrm{GL}_{n}(\mathbb{Q})$ such that $(\phi, f): \Gamma_{0} \curvearrowright X_{0} \longrightarrow \mathrm{GL}_{n}(\mathbb{Q}) \curvearrowright \mathrm{Gr}_{k}\left(\mathbb{Q}_{p}^{n}\right)$ is a homomorphism of permutation groups.

By Lemma 3.11, we may replace $\Gamma_{0} \curvearrowright X_{0}$ with an ergodic subcomponent to suppose that $\phi\left(\Gamma_{0}\right) \subset \mathrm{SL}_{n}(\mathbb{Z})$ is a subgroup of finite index. Shortly, we shall argue that we can suppose that $f\left(X_{0}\right)$ is an ergodic component for the action of $\phi\left(\Gamma_{0}\right)$. However, since our argument is sensitive to timing, we must first perform a simplification which will make the computations at the end of the proof easier.

By Proposition 2.3, we may replace $X_{0}$ with an ergodic subcomponent to suppose that $\Gamma_{0} \curvearrowright X_{0}$ is a principle congruence component. Recall that this means $\Gamma_{0}$ is some principle congruence subgroup $\Gamma_{p^{t}}$ and that $X_{0}$ is equal, modulo a null set, to a $K_{p^{t} \text {-orbit. }}$

5.6. Claim. We may suppose that the domain $X_{0}$ of $f$ is equal, modulo a null set, to the particular ergodic component $Z_{0}=\left(K_{p^{t}}\right) V_{0}$.

Recall that the ergodic component $Z_{0}$ was described in equation (2.2).

Proof of claim. Recall that $\mathrm{SL}_{n}(\mathbb{Z})$ acts transitively on the $K_{p^{t}}$-orbits, so there exists $\gamma \in \mathrm{SL}_{n}(\mathbb{Z})$ such that $\gamma Z_{0}=X_{0}$, modulo a null set. Consider the map $f^{\prime}(x):=f(\gamma x)$. By Lemma 4.1, we always have $x E_{\cong}^{k} \gamma x$, and so $f^{\prime}$ is a Borel reduction from $E_{\cong}^{k}$ to $E_{\mathrm{GL}_{n} \mathbb{Q}}^{k}$. Moreover, it is easily checked that $\left(\phi^{\prime}, f^{\prime}\right)$ is a homomorphism of permutation groups, where $\phi^{\prime}(g)=\phi\left(\gamma g \gamma^{-1}\right)$. Replacing $(\phi, f)$ with $\left(\phi^{\prime}, f^{\prime}\right)$ establishes the claim.

5.7. Claim. We may suppose that $f(x)=x$ for all $x \in X_{0}$.

Proof of claim. Observe that since the $\gamma$ from the last argument satisfies $\gamma \in$ $\mathrm{SL}_{n}(\mathbb{Z})$, we have retained that $\phi\left(\Gamma_{0}\right) \subset \mathrm{SL}_{n}(\mathbb{Z})$. Now, by the ergodicity of $\Gamma_{0} \curvearrowright X_{0}$, we may delete a null subset of $X_{0}$ to suppose that $f\left(X_{0}\right)$ is contained in an ergodic component for the action of $\phi\left(\Gamma_{0}\right)$. By Theorem 2.4 we may suppose that there exists $h \in \mathrm{GL}_{n}(\mathbb{Q})$ such that $f(x)=h x$ for all $x \in X_{0}$. But now, since $h \in \mathrm{GL}_{n}(\mathbb{Q})$, it follows that the identity map on $X_{0}$ is also a Borel reduction from $E_{\cong}^{k}$ to $E_{\mathrm{GL}_{n} \mathbb{Q}}^{k}$. $\dashv$

Before proceeding to the final contradiction, we give a brief outline. If indeed the identity function on $X_{0}$ is a Borel reduction from $E_{\cong}^{k}$ to $E_{\mathrm{GL}_{n}}^{k} \mathbb{Q}$, then whenever $x, g x \in X_{0}$ and $g \in \mathrm{GL}_{n}(\mathbb{Q})$, we will have $x E_{\cong}^{k} g x$. If we additionally suppose that $x, g x \in Z_{0}$, then as we have observed, $x$ and $g x$ each have the canonical complementary subspace $V_{1}=\mathbb{Q}_{p} e_{k+1} \oplus \cdots \oplus \mathbb{Q}_{p} e_{n}$. We shall choose the matrix $g$ so that it acts "nontrivially" on $V_{1}$ and this will contradict that $x E_{\cong}^{k} g x$.

Turning to the details, let $g=\operatorname{diag}(1, \ldots, 1, p)$, where $\operatorname{diag}\left(d_{1}, \ldots, d_{n}\right)$ denotes the diagonal matrix with $a_{i i}=d_{i}$. Using equation (2.2) one easily checks that $g Z_{0} \subset Z_{0}$, and since $X_{0}=Z_{0}$ modulo a null set, we have that $g X_{0}$ is almost contained in $X_{0}$. Together with Lemma 3.9, this implies that we can choose $x \in$ $\operatorname{Fr}\left(\mathrm{PGL}_{n}(\mathbb{Q}) \curvearrowright \operatorname{Gr}_{k}\left(\mathbb{Q}_{p}^{n}\right)\right)$ in such a way that $x, g x \in X_{0} \cap Z_{0}$. Then $x\left(E_{\cong}^{k}\right) g x$, and so Definition 4.9 gives $h \in \mathrm{GL}_{n}(\mathbb{Q})$ such that

$$
h\left(x, L_{0}\right)=\left(g x, L_{0}\right) .
$$


Now, $h x=g x$ and since we have chosen $x$ so that it is not fixed by any element of $\operatorname{PGL}_{n}(\mathbb{Q}) \backslash\{1\}$, there exists $a \in \mathbb{Q}_{p}^{*}$ such that $h=a g$. Since $x, g x \in Z_{0}$, each has the canonical complementary subspace $V_{1}=\mathbb{Q}_{p} e_{k+1} \oplus \cdots \oplus \mathbb{Q}_{p} e_{n}$. Since $h$ is diagonal, it clearly acts on $V_{1}$ via its $(n-k) \times(n-k)$ lower right-hand corner $h^{c}$. It follows that $h\left(x, L_{0}\right)=\left(h x, h^{c} L_{0}\right)$, which (together with (5.8) $)$ implies that $h^{c}$ stabilizes $L_{0}$. But it is readily seen that $\nu_{p}\left(\operatorname{det} h^{c}\right) \equiv 1 \bmod (n-k)$, so Proposition 5.4 implies that $h^{c}$ does not stabilize $L_{0}$, a contradiction.

We next attack the hardest case of Theorem A.

5.9. Theorem (Theorem A, case 2). Suppose that $n \geq 4$ and $2 \leq k<n / 2$. Then $\cong_{n, p}^{k}$ is Borel incomparable with $\cong_{n, p}^{n-k}$.

This is a consequence of the following slightly stronger result.

5.10. Theorem. Suppose that $n \geq 3$, and let $2 \leq k \leq n-1$ and $k \neq n / 2$. Then $E_{\cong}^{k} \not_{B}^{w} \cong n, p$.

Recall from Section 4 that $\cong_{n, p}^{l}$ is Borel bireducible with the orbit equivalence relation induced by the action of $\mathrm{GL}_{n}(\mathbb{Q})$ on $\mathcal{M}(n, p, l)$. In view of the freeness aspects of the proof of Theorem 3.6, one might expect that it would be necessary to work with the action of $\mathrm{PGL}_{n}(\mathbb{Q})$. However, $\mathrm{PGL}_{n}(\mathbb{Q})$ does not act on $\mathcal{M}(n, p, l)$ ! Instead, we must work with the action of $\operatorname{PGL}_{n}(\mathbb{Q})$ on the space $\mathcal{M}^{*}(n, p, l)$ of equivalence classes $[M]=\left\{a M: a \in \mathbb{Q}_{p}^{*}\right\}$ for $M \in \mathcal{M}(n, p, l)$. (This is indeed a standard Borel space, since the equivalence relation on $\mathcal{M}(n, p, k)$ with equivalence classes $[M]$ is smooth.) Let $\left(\cong_{n, p}^{l}\right)^{*}$ denote the orbit equivalence relation induced by the action of $\mathrm{GL}_{n}(\mathbb{Q})$ on $\mathcal{M}^{*}(n, p, l)$.

5.11. Proposition. The equivalence relation $\left(\cong_{n, p}^{l}\right)^{*}$ is Borel bireducible with $\cong_{n, p}^{l}$.

Proof. Since the completion map $\Lambda$ witnesses that $\cong_{n, p}^{l}$ is Borel bireducible with the orbit equivalence relation $E$ induced by the action of $\mathrm{GL}_{n}(\mathbb{Q})$ on $\mathcal{M}(n, p, l)$, it is enough to check that $\left(\cong_{n, p}^{l}\right)^{*}$ is Borel bireducible with $E$. Clearly, the map $M \mapsto[M]$ is a Borel reduction from $E$ to $\left(\cong_{n, p}^{l}\right)^{*}$. Since this map is countable-toone, it admits a Borel section $\sigma$ which is evidently a Borel reduction from $\left(\cong_{n, p}^{l}\right)^{*}$ to $E$.

The advantage of working with the space $\mathcal{M}^{*}(n, p, l)$ is that we can use the following variant of Lemma 3.9 .

5.12. Lemma. Suppose that $f: \operatorname{Gr}_{k}\left(\mathbb{Q}_{p}^{n}\right) \rightarrow \mathcal{M}^{*}(n, p, l)$ is a weak Borel reduction from $E_{\mathrm{SL}_{n} \mathbb{Z}}^{k}$ to $\left(\cong_{n, p}^{l}\right)^{*}$. Then there exists a conull subset $X \subset \mathrm{Gr}_{k}\left(\mathbb{Q}_{p}^{n}\right)$ such that

$$
f(X) \subset \operatorname{Fr}\left(\mathrm{PGL}_{n}(\mathbb{Q}) \curvearrowright \mathcal{M}^{*}(n, p, l)\right) .
$$

Proof. If $[M]=\left[M^{\prime}\right]$, it is clear that $V_{M}=V_{M^{\prime}}$ (see the notation of (4.3)). Hence the map $[M] \mapsto V_{M}$ is well-defined, and we may consider the function $\bar{f}: \operatorname{Gr}_{k}\left(\mathbb{Q}_{p}^{n}\right) \rightarrow \operatorname{Gr}_{l}\left(\mathbb{Q}_{p}^{n}\right)$ given by $\bar{f}(x)=V_{f(x)}$. Clearly, $\bar{f}$ is a weak Borel reduction from $E_{\mathrm{SL}_{n} \mathbb{Z}}^{k}$ to $E_{\mathrm{GL}_{n} \mathbb{Q}}^{l}$. We claim that there exists a conull subset $X \subset \operatorname{Gr}_{k}\left(\mathbb{Q}_{p}^{n}\right)$ such that

$$
\bar{f}(X) \subset \operatorname{Fr}\left(\mathrm{PGL}_{n}(\mathbb{Q}) \curvearrowright \mathrm{Gr}_{l}\left(\mathbb{Q}_{p}^{n}\right)\right) .
$$

If this is not the case, then Theorem 3.9 implies that there exists a conull subset $X^{\prime} \subset \operatorname{Gr}_{l}\left(\mathbb{Q}_{p}^{n}\right)$ such that $\bar{f}\left(X^{\prime}\right)$ is contained in a single $\mathrm{GL}_{n}(\mathbb{Q})$-orbit of $\mathrm{Gr}_{l}\left(\mathbb{Q}_{p}^{n}\right)$. 
Since $f$ is countable-to-one, it follows that $f\left(X^{\prime}\right)$ is contained in a countable set, contradicting that the Haar measure is nonatomic.

Now, for $x \in X$, we have that $\bar{f}(x) \in \operatorname{Fr}\left(\mathrm{PGL}_{n}(\mathbb{Q}) \curvearrowright \mathrm{Gr}_{l}\left(\mathbb{Q}_{p}^{n}\right)\right)$. This means by definition that $1 \neq g \in \mathrm{PGL}_{n}(\mathbb{Q})$ implies $g V_{f(x)} \neq V_{f(x)}$. Clearly, $g V_{f(x)}=V_{g f(x)}$, and so we have $V_{g f(x)} \neq V_{f(x)}$. It follows that $g f(x) \neq f(x)$, which means that $f(x) \in \operatorname{Fr}\left(\mathrm{PGL}_{n}(\mathbb{Q}) \curvearrowright \mathcal{M}^{*}(n, p, l)\right)$, as desired.

We extend the notion of type to $\mathcal{M}^{*}(n, p, l)$ by letting type $([M])=\operatorname{type}(L)$, where $M=(V, L)$. This is well-defined, as $a M=(V, a L)$ and we have already observed that the type of $L$ depends only on its class. The following fact is the last we shall need in the proof of Theorem 5.10.

5.13. Proposition. The group $\mathrm{SL}_{n}\left(\mathbb{Z}_{p}\right)$ acts in a type-preserving fashion on $\mathcal{M}^{*}(n, p, l)$.

Proof. We must show that whenever $g \in \mathrm{SL}_{n}\left(\mathbb{Z}_{p}\right)$ and $g(V, L)=\left(V^{\prime}, L^{\prime}\right)$, we have that type $(L)=\operatorname{type}\left(L^{\prime}\right)$. First suppose that $V=V^{\prime}=V_{0}$, where $V_{0}=\mathbb{Q}_{p} e_{1} \oplus$ $\cdots \oplus \mathbb{Q}_{p} e_{l}$. Then, in particular, $g$ fixes $V_{0}$. Letting $g^{c}$ denote the $(n-l) \times(n-l)$ lower right-hand corner of $g$, we can argue as in the proof of Proposition 4.6 that $g\left(V_{0}, L\right)=\left(V_{0}, g^{c} L\right)$ and so $L^{\prime}=g^{c} L$. But $g^{c} \in \mathrm{GL}_{n-l}\left(\mathbb{Z}_{p}\right)$, and so Proposition 5.4 implies that type $\left(L^{\prime}\right)=\operatorname{type}(L)$.

Also as in the proof of Proposition 4.6, this special case can be translated to establish the result in the general case.

Proof of Theorem 5.10, Suppose that $f: \operatorname{Gr}_{k}\left(\mathbb{Q}_{p}^{n}\right) \rightarrow \mathcal{M}^{*}(n, p, n-k)$ is a weak Borel reduction from $E_{\cong}^{k}$ to $\left(\cong_{n, p}^{n-k}\right)^{*}$. Then clearly $f$ is a weak Borel reduction from $E_{\mathrm{SL}_{n} \mathbb{Z}}^{k}$ to $\left(\cong_{n, p}^{n-k}\right)^{*}$. Applying the arguments of Theorem 3.6 (and substituting Lemma 5.12 instead of Lemma 3.9 to define a cocycle), we may suppose that there exists an ergodic component $\Gamma_{0} \curvearrowright X_{0}$ for $\mathrm{SL}_{n}(\mathbb{Z}) \curvearrowright \mathrm{Gr}_{k}\left(\mathbb{Q}_{p}^{n}\right)$, and a homomorphism $\phi: \Gamma_{0} \rightarrow \mathrm{GL}_{n}(\mathbb{Q})$ such that

$$
(\phi, f): \Gamma_{0} \curvearrowright X_{0} \longrightarrow \mathrm{GL}_{n}(\mathbb{Q}) \curvearrowright \mathcal{M}^{*}(n, p, n-k)
$$

is a homomorphism of permutation groups.

Since the map $[M] \rightarrow V_{M}$ is $\mathrm{GL}_{n}\left(\mathbb{Q}_{p}\right)$-preserving, the composition $\bar{f}: x \mapsto V_{f(x)}$ makes

$$
(\phi, \bar{f}): \Gamma_{0} \curvearrowright Z_{0} \longrightarrow \mathrm{GL}_{n}(\mathbb{Q}) \curvearrowright \mathrm{Gr}_{n-k} \mathbb{Q}_{p}^{n}
$$

into a homomorphism of permutation groups. By Lemma 3.11, we may replace $\Gamma_{0} \curvearrowright X_{0}$ with an ergodic subcomponent to suppose that $\operatorname{im}(\phi) \subset \mathrm{SL}_{n}(\mathbb{Z})$. As in the proof of Theorem 5.2 we make the following simplifications.

5.14. Claim. We may suppose that $\Gamma_{0}=\Gamma_{p^{t}}$ is a principle congruence subgroup and that $X_{0}$ is equal, modulo a null set, to the particular component $Z_{0}=\left(K_{p^{t}}\right) V_{0} . \dashv$

5.15. Claim. We may suppose that $\bar{f}(x)=x^{\perp}$ for all $x \in X_{0}$.

Proof of claim. As with the last time, the hypotheses of Theorem 2.4 are satisfied, and we may suppose that there exists $h \in \mathrm{GL}_{n}(\mathbb{Q})$ such that $\phi(\gamma)=\chi_{h} \circ(-T)$ and $\bar{f}(x)=h^{-1} x^{\perp}$. Hence, replacing $f$ by $h f$ and $\phi$ with $-T$, we obtain the desired result.

Observe that in the last argument, we have retained the property that $(\phi, f)$ is a homomorphism of permutation groups such that $\operatorname{im}(\phi) \subset \mathrm{SL}_{n}(\mathbb{Z})$. For the next 
claim, recall that we have defined that type $([M])$ is the type of any $L$ such that $(V, L) \in[M]$.

5.16. Claim. We can suppose that there is a fixed $0 \leq t<k$ such that type $(f(x))=t$ for all $x \in X_{0}$.

Proof of claim. Let $F\left(\mathcal{M}^{*}(n, p, n-k)\right)$ denote the standard Borel space of closed subsets of $\mathcal{M}^{*}(n, p, n-k)$ (where $\mathcal{M}(n, p, n-k)$ is considered with the quotient topology induced by the map $M \mapsto[M])$. Since $\phi\left(\Gamma_{0}\right) \subset \mathrm{SL}_{n}(\mathbb{Z})$, we clearly have that the map $X_{0} \rightarrow F\left(\mathcal{M}^{*}(n, p, n-k)\right)$ given by

$$
x \mapsto\left(\mathrm{SL}_{n} \mathbb{Z}_{p}\right) f(x)
$$

is $\Gamma_{0}$-invariant. By ergodicity of $\Gamma_{0} \curvearrowright X_{0}$, we may suppose that $f\left(X_{0}\right)$ is contained in a fixed $\mathrm{SL}_{n}\left(\mathbb{Z}_{p}\right)$-orbit. Hence, the claim follows from Proposition 5.13

The proof now concludes in a manner similar to that of Theorem 5.2, Roughly speaking, we shall choose generic $x, g x \in X_{0}$ so as to guarantee that $f(g x)=$ $g^{-T} f(x)$. We shall also select $g \in \mathrm{GL}_{n}(\mathbb{Q})$ so that $g^{-T}$ fails to preserve lattice types on $f\left(X_{0}\right)$, contradicting the last claim.

More specifically, let $g=\operatorname{diag}(1 / p, 1, \ldots, 1)$, where $\operatorname{diag}\left(d_{1}, \ldots, d_{n}\right)$ denotes the diagonal matrix with $a_{i i}=d_{i}$. Arguing as in the proof of Theorem [5.1] we can choose $x \in X_{0}$ such that $g x \in X_{0}$ and $x^{\perp} \in \operatorname{Fr}\left(\mathrm{PGL}_{n}(\mathbb{Q}) \curvearrowright \mathrm{Gr}_{n-k} \mathbb{Q}_{p}^{n}\right)$. Now, both $x$ and $g x$ have the canonical complementary subspace $V_{1}=\mathbb{Q}_{p} e_{k+1} \oplus \cdots \oplus \mathbb{Q}_{p} e_{n}$. Since $g$ acts on $V_{1}$ by the identity map, we clearly have $g\left(x, L_{0}\right)=\left(g x, L_{0}\right)$, and so $g$ witnesses that $x E_{\cong}^{k} g x$. It follows that $f(x)\left(\cong_{n, p}^{n-k}\right)^{*} f(g x)$, and hence there exists $h \in \mathrm{GL}_{n}(\mathbb{Q})$ such that $h f(x)=f(g x)$. Then $h \bar{f}(x)=\bar{f}(g x)$, so now

$$
h x^{\perp}=h \bar{f}(x)=\bar{f}(g x)=(g x)^{\perp}=g^{-T} x^{\perp} .
$$

Since we have chosen $x^{\perp}$ so that it is not fixed by any element of $\mathrm{PGL}_{n}(\mathbb{Q}) \backslash\{1\}$, there exists $a \in \mathbb{Q}_{p}^{*}$ such that $h=a g^{-T}=\operatorname{diag}(a p, a, \ldots, a)$ (see Figure 1).

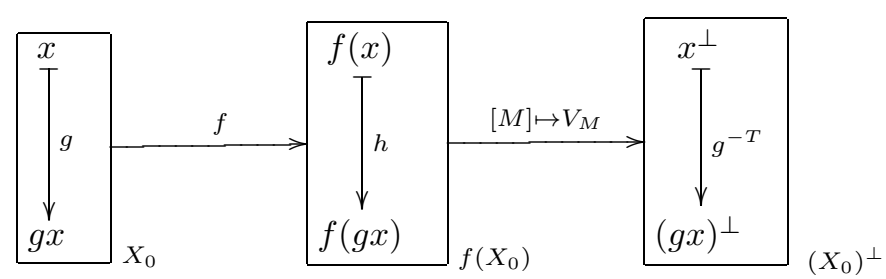

Figure 1. We have guaranteed that $f(g x)=h f(x)$, where $h=a g^{-T}$.

Finally, $x^{\perp},(g x)^{\perp}$ each have the canonical complementary subspace $V_{0}=\mathbb{Q}_{p} e_{1} \oplus$ $\cdots \oplus \mathbb{Q}_{p} e_{k}$. Letting $h^{c}$ denote the upper left-hand corner of $h$, we have $\nu_{p}\left(h^{c}\right) \equiv 1$ $\bmod k$, and so by Proposition 5.4, $h^{c}$ acts in a type-altering fashion on $\mathcal{L}\left(V_{0}\right)$. But we have arranged for type $(f(x))=$ type $(f(g x))$, a contradiction.

The second part of Theorem B follows immediately. 
5.17. Theorem (Theorem B, part 2). If $n \geq 3$ and $k \leq n-2$, then $E_{\mathrm{GL}_{n}}^{k} \mathbb{Q} \mathbb{Z}_{B}^{w} E_{\cong}^{k}$.

Proof. The orthogonal complement map witnesses that $E_{\mathrm{GL}_{n} \mathbb{Q}}^{k}$ is Borel bireducible with $E_{\mathrm{GL}_{n} \mathbb{Q}}^{n-k}$. Hence, if $E_{\mathrm{GL}_{n} \mathbb{Q}}^{k} \leq_{B}^{w} E_{\cong}^{k}$, then there exists a weak Borel reduction $f$ from $E_{\mathrm{GL}_{n} \mathbb{Q}}^{n-k}$ to $E_{\cong}^{k}$. Clearly, $f$ is also a weak Borel reduction from $E_{\cong}^{n-k}$ to $E_{\cong}^{k}$, contradicting Theorem 5.10 .

The keen-eyed reader will have noticed that Theorem A is as yet incomplete.

5.18. Theorem (Theorem A, case 3). If $n \geq 3$, then $E_{\cong}^{1} \mathbb{L}_{B} E_{\cong}^{n-1}$.

Proof. By [Tho02, Theorem 4.4], for groups $A, B \in R(n, p, n-1)$ we have that $A$ is quasi-isomorphic to $B$ iff $A$ is isomorphic to $B$. In particular, $\cong_{n, p}^{n-1}$ is Borel bireducible with $\sim_{n, p}^{n-1}$, and it follows that $E_{\cong}^{n-1}$ is Borel bireducible with $E_{\mathrm{GL}_{n}}^{n-1} \mathbb{Q}$. Again using the orthogonal complement map, $E_{\mathrm{GL}_{n} \mathbb{Q}}^{n-1}$ is Borel bireducible with $E_{\mathrm{GL}_{n} \mathbb{Q}}^{1}$, and so we have established that the right-hand side $E_{\cong}^{n-1}$ is Borel bireducible with $E_{\mathrm{GL}_{n} \mathbb{Q}}^{1}$. Hence, the result follows from Theorem B, part 1 .

\section{REFERENCES}

[Ada02] Scot Adams. Containment does not imply Borel reducibility. In Set theory (Piscataway, NJ, 1999), volume 58 of DIMACS Ser. Discrete Math. Theoret. Comput. Sci., pages 1-23. Amer. Math. Soc., Providence, RI, 2002. MR1903846 (2003j:03059)

[AK00] Scot Adams and Alexander S. Kechris. Linear algebraic groups and countable Borel equivalence relations. J. Amer. Math. Soc., 13(4):909-943 (electronic), 2000. MR1775739 (2001g:03086)

[Bro89] Kenneth S. Brown. Buildings. Springer-Verlag, New York, 1989. MR969123 (90e:20001)

[FM77] Jacob Feldman and Calvin C. Moore. Ergodic equivalence relations, cohomology, and von Neumann algebras. I. Trans. Amer. Math. Soc., 234(2):289-324, 1977. MR0578656 $(58: 28261 \mathrm{a})$

[FS89] Harvey Friedman and Lee Stanley. A Borel reducibility theory for classes of countable structures. J. Symbolic Logic, 54(3):894-914, 1989. MR1011177 (91f:03062)

[Fuc73] László Fuchs. Infinite abelian groups. Vol. II. Academic Press, New York, 1973. Pure and Applied Mathematics. Vol. 36-II. MR0349869 (50:2362)

[Hjo99] Greg Hjorth. Around nonclassifiability for countable torsion free abelian groups. In Abelian groups and modules (Dublin, 1998), Trends Math., pages 269-292, Birkhäuser, Basel, 1999. MR1735575 (2001c:20113)

[HK96] Greg Hjorth and Alexander S. Kechris. Borel equivalence relations and classifications of countable models. Ann. Pure Appl. Logic, 82(3):221-272, 1996. MR1423420 (99m:03073)

[HT06] Greg Hjorth and Simon Thomas. The classification problem for $p$-local torsionfree abelian groups of rank two. J. Math. Log., 6(2):233-251, 2006. MR.2317428 (2008i:03053)

[Ioa07a] Adrian Ioana. Cocycle superrigidity for profinite actions of property $(\mathrm{T})$ groups. Submitted, and available at arXiv:0805.2998v1 [math.GR], 2007.

[Ioa07b] Adrian Ioana. Some rigidity results in the orbit equivalence theory of non-amenable groups. Ph.D. thesis, UCLA, 2007.

[Lub03] Alex Lubotzky. What is property $(\tau)$ ? Notices Amer. Math. Soc. 52(6):626-627, 2005. MR 2147485

[Ser03] Jean-Pierre Serre. Trees. Springer Monographs in Mathematics. Springer-Verlag, Berlin, 2003. Translated from the French original by John Stillwell, Corrected 2nd printing of the 1980 English translation. MR607504 (82c:20083)

[Tho02] Simon Thomas. The classification problem for $p$-local torsion-free abelian groups of finite rank. Unpublished preprint, 2002.

[Tho03a] Simon Thomas. The classification problem for torsion-free abelian groups of finite rank. J. Amer. Math. Soc., 16(1):233-258 (electronic), 2003. MR.1937205|(2004b:20081) 
[Tho03b] Simon Thomas. Superrigidity and countable Borel equivalence relations. Ann. Pure Appl. Logic, 120(1-3):237-262, 2003. MR.1949709 (2004e:03081)

[Zim84] Robert J. Zimmer. Ergodic theory and semisimple groups, volume 81 of Monographs in Mathematics. Birkhäuser Verlag, Basel, 1984. MR776417 (86j:22014)

Mathematics Program, The Graduate Center of The City University of New York, 365 Fifth Avenue, New York, New York 10016

E-mail address: scoskey@nylogic.org

$U R L:$ http://math.rutgers.edu/ scoskey

Current address: York University and The Fields Institute, 222 College Street, Toronto, Ontario, Canada M5S 2N2 